\section{A Slide Rule for Haematological Calculations}

\author{
J. W. NICHOLAS \\ From the Pathology Laboratory, Essex County Hospital, \\ Colchester
}

\section{(RECEIVED FOR PUBLICATION DECEMBER 22, 1956)}

The calculation of the mean corpuscular volume (M.C.V.), mean corpuscular haemoglobin (M.C.H.), mean corpuscular haemoglobin concentration (M.C.H.C.), and the colour index (C.I.), where this is still used, involves only the simplest arithmetic; nevertheless in routine laboratory practice it occasions a considerable waste of time, and errors are fairly frequent.

Wintrobe (1951) gives a nomogram for these calculations, but nomograms are not very convenient to use, and his has two different scales for haemoglobin which are liable to cause confusion. It is also not immediately apparent what arithmetical manipulation is being performed by the nomogram. A slide rule is a practicable alternative, but haematological technicians usually seem reluctant to use one. There are three disadvantages associated with the conventional slide rule. First, the normal values for colour index and mean corpuscular volume both come at or near the end of the scale; resetting of the rule is therefore required frequently. Secondly, the unskilled user is apt to read the quotient of a division at the wrong end of the rule, thus getting the reciprocal of the required number (the position of the decimal point being of course ignored). This is particularly dangerous in view of the normal values expected. From Table $I$ it will be seen that in no case is the reciprocal of a normal value so obviously impossible as to be rejected. Thirdly, a separate manipulation has to be carried out for each individual division.

\section{Description of Instrument}

An instrument has been devised which avoids all the above disadvantages, and which, it is claimed, enables
TABLE I

PROBABLE ERRORS IN READING HAEMATOLOGICAE VALUES FROM CONVENTIONAL SLIDE RULE

\begin{tabular}{|c|c|c|c|c|}
\hline Value & & Normal & Reciprocal & $\begin{array}{l}\text { Liable to } \\
\text { be Read as }\end{array}$ \\
\hline $\begin{array}{l}\text { M.C.V. } \\
\text { M.C.H. } \\
\text { M.C.H.C. } \\
\text { C.I. . . }\end{array}$ & $\begin{array}{l}\ldots \\
\cdots \\
\cdots\end{array}$ & $\begin{array}{l}86 \\
29.5 \\
34 \\
1.0\end{array}$ & $\begin{array}{l}0.0116 \\
0.0339 \\
0.0294 \\
1.0\end{array}$ & $\begin{array}{r}116 \\
33.9 \\
29.4 \\
1.0\end{array}$ \\
\hline
\end{tabular}

anyone without prior knowledge of the use of a slidgs rule to calculate a full set of "absolute values" in tक. matter of seconds with minimal chance of error. I\$ addition, a scale is provided which can be used as $\delta$ calibration chart for a colorimeter used for haemoiv globinometry. The rule is shown in Fig. 1. It will beu seen that there are five main scales, marked A, B, Co $D, E$, and shorter scales, F, G, H, J, on the right-han $\phi$ ends of certain of the main scales. Scales $B$ and $E$. (and $F, G$, and $J$ on their right-hand ends) are on the stock of the rule and are immovable; scales A, C, and and $\mathrm{H}$ at the right hand end of $\mathrm{D}$ are movable. The purpose of the various scales is shown in Table II.

TABLE II

DETAILS OF SCALES

\begin{tabular}{|c|c|c|}
\hline Scale & Unit & Range \\
\hline \multirow[t]{2}{*}{$\begin{array}{l}\mathbf{A} \\
\mathbf{B} \\
\mathbf{C} \\
\mathbf{D} \\
\mathbf{E} \\
\mathbf{F} \\
\mathbf{G} \\
\mathbf{H} \\
\mathbf{J}\end{array}$} & 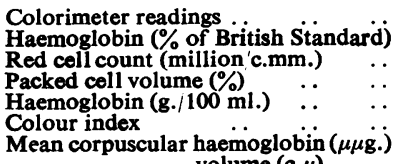 & \multirow{2}{*}{$\begin{array}{c}3-100 \\
10-160 \\
0 \cdot 5-10 \\
5-100 \\
1 \cdot 5-26 \\
0 \cdot 35-1 \cdot 7 \\
10-50 \\
40-180 \\
10-50\end{array}$} \\
\hline & 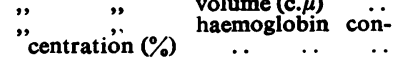 & \\
\hline & Method of Use & \\
\hline
\end{tabular}
globin and most of its derivatives used for haemog globinometry follow Beer's law fairly strictly and with 8 suitable photoelectric colorimeter or with a spectro photometer a linear calibration curve, passing through the origin, will be obtained. The rule can be calibrated for such an instrument by simply moving Scale A untib

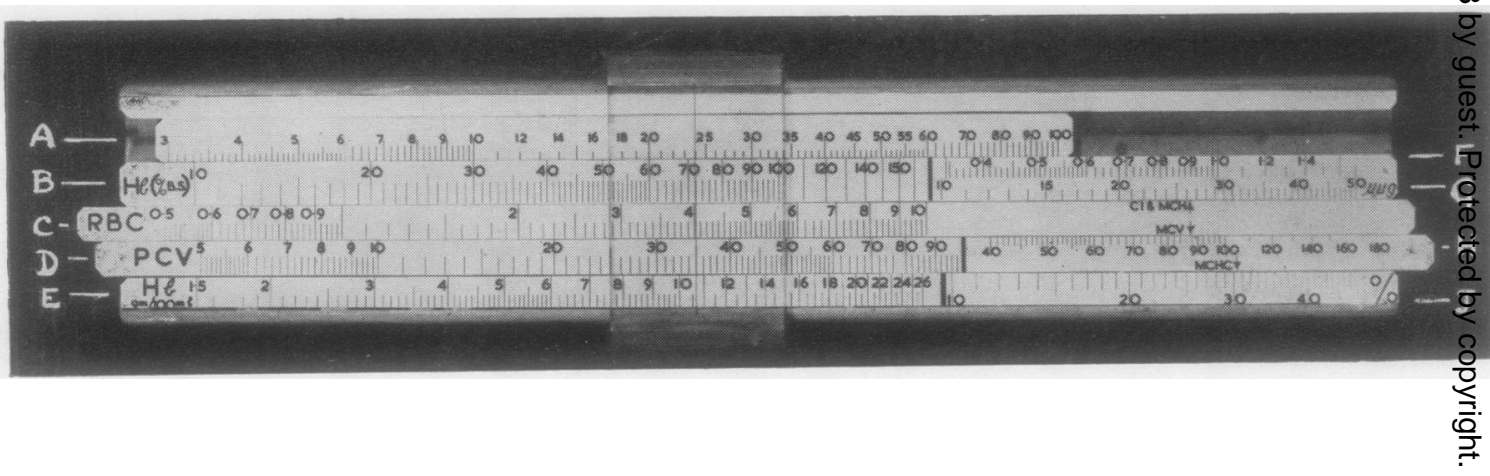


the reading for a standard blood solution on it is in line with the known haemoglobin concentration in $\%$ of British Standard or g. $/ 100 \mathrm{ml}$. as desired on Scale B or Scale E. Scale A can be reversed from end to end so that it reads in reciprocals for the benefit of any laboratory which may be using such a method as the Newcomer with cup and plunger colorimeter. It can also be turned upside down to provide a matt white surface on which can be drawn a special scale if the colorimeter calibration should be found to be significantly nonlinear. This setting is made whenever the calibration of the instrument is checked and is not disturbed thereafter. Scale $\mathbf{A}$ is therefore made stiff in its slide, and is shorter than the body of the rule so as not to be displaced by knocking the ends.

Use of the Slide Rule.-This is best seen by a practical example. Fig. 1 shows the rule set for this.

(1) Haemoglobinometer Reading. -24 arbitrary units. Set cursor to 24 .

(2) Red Cell Count.-4.05 million/c.mm. Move Sca'e $\mathrm{C}$ to bring 4.05 under cursor line.

(3) Packed Cell Volume.-35\%. Move Scale D to bring 35 under cursor line.

These are the only manipulations to be performed and all required values may now be read directly in their correct units and with correct positioning of the decimal point. Thus:

(4) Haemoglobin is read on Scale B or E under the cursor line $=72 \%$ B.S. $=10.6 \mathrm{~g} .1100 \mathrm{ml}$.

(5) Colour index and mean corpuscular haemoglobin are read on Scales $F$ and $G$ over the combined index mark (C.I. and M.C.H.) on the slide immediately below them. (The fact that these scales are both fixed and read by a single index mark should help to emphasize the linear relationship between the two.)

$$
\begin{aligned}
& \text { C.I. }=0.9 \\
& \text { M.C.H. }=26.2 \mu \mu \mathrm{g} .
\end{aligned}
$$

(6) Mean corpuscular volume on Scale $H$ below the index mark (M.C.V.) on the slide above.

$$
\text { M.C.V. }=87 \text { c. } \mu \text {. }
$$

(7) Mean corpuscular haemoglobin concentration on Scale $J$ below the index mark (M.C.H.C.) on the slide above.

$$
\text { M.C.H.C. }=30.5 \% \text {. }
$$

It is necessary to meet one possible objection, namely, that students using the instrument might not realize what they are in fact doing. Simple logarithmic scales are used throughout and it should be obvious that the determination of the haemoglobin from the colorimeter reading is simply a matter of direct proportion and that the other values are all the quotients of divisions, the divisor in each case being the value on the scale which bears the index mark for reading the quotient.

\section{Summary}

A slide rule is described suitable for the rapid calculation of mean corpuscular volume, mean corpuscular haemoglobin, mean corpuscular haemoglobin concentration, and colour index. It also incorporates a scale which can be pre-set to form the calibration of a photoelectric or other haemoglobinometer. The instrument can be used by a worker who has no previous experience of a slide rule, and the chance of wrong readings is negligible.

I am indebted to Messrs. E. N. Mason \& Sons, of Arclight Works, Colchester, for their most valuable help in making the prototype.

The rule will be commercially available from Messrs. Camlab (Glass) Ltd., 50, Burleigh Street, Cambridge.

\section{REFERENCE}

Wintrobe, M. M. (1951). Clinical Haematology. Henry Kimpton, London (Fig. 62, p. 330). 